\title{
Surveillance for Hepatocellular Carcinoma in a Medicaid Cirrhotic Population
}

\author{
Lena B. Palmer, MD MSCR ${ }^{1}$, Michael D. Kappelman, MD, MPH ${ }^{2}$, Robert S. Sandler, MD, \\ $\mathrm{MPH}^{4}$, and Paul H. Hayashi, MD, MPH ${ }^{5}$
}

${ }^{1}$ Division of Gastroenterology, Hepatology, \& Nutrition; Department of Medicine, Loyola University Chicago Stritch School of Medicine; Maywood, IL; Ipalmer@lumc.edu 2Division of Gastroenterology and Hepatology, Department of Pediatrics, University of North Carolina, Chapel Hill, North Carolina, michael_kappelman@med.unc.edu ${ }^{4}$ Division of Gastroenterology and Hepatology, Department of Medicine, University of North Carolina, Chapel Hill, North Carolina, robert_sandler@med.unc.edu ${ }^{5}$ Division of Gastroenterology and Hepatology, Department of Medicine, University of North Carolina, Chapel Hill, North Carolina, paul_hayashi@med.unc.edu

\section{Abstract \\ Goals-To estimate hepatocellular carcinoma surveillance in the Medicaid cirrhotic population. \\ Background-Most studies predate 2005 AASLD surveillance recommendations and do not examine the primary target population, cirrhotics.}

Study-For 2006-2007 we identified adults with at least one cirrhosis International Classification of Disease code and 15 months of continuous enrollment in North Carolina Medicaid, recording claims for abdominal ultrasound, computed tomography, magnetic resonance imaging, and alphafetoprotein testing. We used multi-variable logistic regression to identify factors independently associated with imaging.

\begin{abstract}
Results-Five-thousand sixty one subjects $(5,061)$ were identified: mean age 54 years, $54 \%$ male, 35\% African-American, 56\% White. Cirrhosis risk factors were alcohol (59\%), hepatitis C (30\%), hepatitis B (4\%), other (18\%), and unknown (24\%). Only $26 \%$ had at least one imaging test. Just $12 \%$ of those not hospitalized or seen in an emergency department had any imaging. Care in an academic facility, younger age, female gender, viral hepatitis, and Medicare coinsurance were positively associated with imaging. Twenty-one percent saw a gastroenterologist
\end{abstract}

\footnotetext{
Corresponding Author (requests for reprints should be directed to author below): Lena Palmer, MD, MSCR, Loyola University Chicago Stritch School of Medicine, Department of Medicine, Division of Gastroenterology, Hepatology \& Nutrition, 2160 S. $1^{\text {st }}$ Avenue, Building 54 Room 167, Maywood, IL 60153, Phone: (708) 216-0464, Fax: (708) 216-4113, lpalmer@lumc.edu.

Conflicts of Interest: No authors have conflicts of interest to disclose.

Specific author contributions:

Dr. Palmer participated in the conception and design of the study, data preparation, data analysis, and manuscript preparation. She was involved in final document editing and has approved the final draft submitted.

Dr. Kappelman participated in the conception and design of the study, data analysis, and manuscript preparation. He was involved in final document editing and has approved the final draft submitted.

Dr. Sandler participated in the conception and design of the study. He was involved in final document editing and has approved the final draft submitted.

Dr. Hayashi participated in the conception and design of the study, data analysis, and manuscript preparation. He was involved in final document editing and has approved the final draft submitted.

This is a PDF file of an unedited manuscript that has been accepted for publication. As a service to our customers we are providing this early version of the manuscript. The manuscript will undergo copyediting, typesetting, and review of the resulting proof before it is published in its final citable form. Please note that during the production process errors may be discovered which could affect the content, and all legal disclaimers that apply to the journal pertain.
} 
which increased the odds of undergoing imaging [Odds Ratio (O.R.) 2.81, 95\% Confidence Interval (C.I.) 2.32, 3.41] while primary care visits did not (O.R. 0.94, 95\% C.I. 0.76, 1.16).

Conclusions-Only a quarter of North Carolina Medicaid cirrhotics had abdominal imaging over a 15 month period, and many tests may have occurred without surveillance intent.

Gastroenterology visits nearly tripled the odds of imaging, but primary care visits had no effect. Efforts to improve surveillance rates in cirrhotic patients should target primary care and increased access to sub-specialty care.

\section{Keywords}

screening; quality of care; guidelines; administrative data; health services

\section{Introduction}

Hepatocellular carcinoma (HCC) is a leading cause of death in patients with cirrhosis. $(1,2)$ Moreover, the rate of $\mathrm{HCC}$ in patients with cirrhosis is rising $(3,4)$ and among men, HCC carries the fastest growing death rate of all cancers in the US.(5) In 2005, the American Association for the Study of Liver Diseases (AASLD) recommended HCC surveillance every 6 to 12 months based on randomized controlled trial evidence that surveillance could reduce mortality. $(3,6)$ Therefore, it is important to examine baseline surveillance rates and variation for this aggressive malignancy.

Several studies have provided estimates of HCC surveillance prior to the 2005 guidelines, (7-9) one in the target population of patients with cirrhosis.(10) However, most estimates were based on gastroenterologist's self-reported surveillance rates, estimates from patients who ultimately developed HCC, and older populations who may undergo less surveillance due to age-related risks of morbidity from HCC therapies.

No studies have examined the Medicaid population that typically has less expendable income and less access to health care services. Also, all previous analyses mentioned have examined surveillance in years prior to publication of the 2005 AASLD guidelines. Therefore, we used an administrative health claims database to examine the utilization of abdominal imaging as a surrogate for surveillance in a Medicaid population of adult cirrhotics without prior diagnosis of HCC, limited to data after 2005.

\section{Materials and Methods}

\section{Study Design}

We ascertained performance of abdominal imaging and AFP testing among North Carolina (NC) Medicaid members with at least 15 months of continuous enrollment during 2006-2007. Although surveillance is recommended for cirrhotic individuals every 6-12 months,(3) we expanded the observation period to 15 months in order to capture imaging events just outside of this range.

\section{Data Source}

The North Carolina Cost and Quality Initiative (C.C.Q.I.) database contains de-identified billing information, including diagnostic (International Classification of Disease, I.C.D.-9) and procedural (Current Procedural Terminology, C.P.T.) codes, clinic visits, provider codes, hospitalization codes, and basic demographics for the North Carolina (NC) Division of Medical Assistance or NC Medicaid. All billing information was obtained for covered subjects. This included data from Medicare dual-eligible subjects but did not include subjects with private insurance obtained individually or through an employer. The authors 
and administrators of the C.C.Q.I. received approval from the NC Department of Medical Assistance prior to initiation and publication of this work.

\section{Study Population}

We included all individuals over the age of 18 with 1 ) at least one distinct recording of either I.C.D.-9 code 571.2 (alcoholic cirrhosis of liver) or I.C.D.-9 code 571.5 (cirrhosis of liver without mention of alcohol), and 2) at least 15 months of continuous Medicaid enrollment during 2006-2007. The 15 months of enrollment occurred after the index diagnostic code. When subjects had codes for both alcoholic and non-alcoholic cirrhosis, they were classified as having alcoholic cirrhosis. Patients were excluded if they also had HCC (155.0) or procedure codes indicative of HCC treatment (e.g. hepatic resection, ablation, transplantation) prior to the first cirrhosis diagnostic code. Risk factors for cirrhosis were also identified and included chronic hepatitis B (070.52), chronic hepatitis C (070.54), hemochromatosis (275), alpha1-antitrypsin deficiency (273.4), other chronic non-alcoholic liver disease (571.8), other chronic active hepatitis (571.49), primary biliary cirrhosis (571.6), cholangitis (576.1) and alcohol abuse (571.0-571.3; 303). There are no dedicated I.C.D.-9 codes for auto-immune liver disease, non-alcoholic fatty liver disease, or primary sclerosing cholangitis. The presence (or absence) of risk factors was determined by ICD-9 codes occurring during the 15 months of enrollment.

\section{Surveillance Definition}

Our primary outcome was receipt of at least one radiographic exam that could be used for HCC surveillance over the 15-month observation period. This included abdominal ultrasound, which is the method preferred by the AASLD.(3) However, the sensitivity of ultrasound in detecting small focal liver lesions in patients with cirrhosis is lower than either computed tomography (CT) or magnetic resonance imaging (MRI),(11) possibly leading some individual providers to choose these modalities for surveillance despite the AASLD guidelines. Therefore, we ascertained all instances of abdominal ultrasound [complete abdominal ultrasound (C.P.T. 76700), or limited abdominal ultrasound (C.P.T. 76705)], abdominal computed tomography (CT) with or without contrast (C.P.T. 74170), or magnetic resonance imaging (MRI) of the abdomen with or without contrast (C.P.T. 74183). As it is not considered a stand-alone surveillance test,(3) alpha-fetoprotein (AFP) serum testing (C.P.T. 82105) was recorded but not included in the primary outcome. Administrative data does not contain information regarding the indication for exams, and we could not differentiate between true surveillance exams and diagnostic tests. Therefore, we refer to the primary outcome as receipt of "imaging" and not "surveillance".

\section{Variables Examined}

In addition to risk factors for cirrhosis, we analyzed demographic data such as age, gender, race, and county of residence as well as health services characteristics such as visits to primary care and gastroenterology, care in an academic facility, Medicare co-insurance status, rural versus urban residence, emergency department encounters, and hospitalizations. Race/ethnicity was defined by NC Medicaid and was examined to investigate potential disparities in care. Visits to primary care or gastroenterology were outpatient episodes of care classified by the provider's self-defined specialty. Care in an academic facility was defined as any episode of care in one of the five academic teaching hospitals in North Carolina. Rural versus urban county of residence was based on the North Carolina Rural Databank designation.(12) Information on number and type of medical co-morbidities was collected to calculate the Deyo modification of the Charlson comorbidity index.(13) This was used to adjust for a subject's general clinical severity. 


\section{Subgroup analyses}

Subgroup analyses excluding subjects over 70 years old, those with at least two or three distinct I.C.D.-9 codes for cirrhosis, and those who were not hospitalized or seen in an emergency department (E.D.) were performed. Many cirrhotics over 70 are likely ineligible for HCC treatments such as chemoembolization or transplantation, thus obviating the need for surveillance. Use of multiple I.C.D. cirrhosis codes may reduce much of the inadvertent misclassification of non-cirrhotics as cirrhotic. The third subgroup analysis was to evaluate the potential for miss-classification of surveillance versus diagnostic exams, as we hypothesized that exams ordered for patients who never required hospitalization or an E.D. visit would have a higher likelihood of being a surveillance exam. Given that the source of data was administrative claims, information on severity of cirrhosis and specific laboratory or diagnostic testing results was not available.

Two additional subgroup analyses excluded those subjects who developed HCC during the time of the study to avoid capturing exams that were performed in evaluation of cancer. We also examined utilization limited to ultrasound use only, the preferred surveillance modality.

\section{Statistical Analysis}

Standard univariate statistics were performed, including calculations of means, medians, proportions, ranges and interquartile ranges. Continuous variables were examined in simple continuous form as well as categorical forms in order to determine the most valid and precise coding structure. Bivariate statistics (Pearson's chi-square, Wilcoxon rank-sum, or ttests based on variable type) were used to assess for statistical associations between the receipt of imaging and the exposures of interest. We performed multivariable logistic regression to determine independent factors associated with imaging and adjusted for comorbidities and frequency of cirrhosis diagnostic codes as a proxy of health services utilization (primary care visits were not used as this was considered a variable of interest). As each exposure was considered of potential interest, all risk factors were retained in the final model regardless of the significance in bivariate analysis. Age was significant in a linear fashion in bivariate testing in all ways examined. Therefore, it was left as a continuous variable in the final model. Stata statistical software (Stata Ver. 10.0, College Station, Texas) was used for all analyses. The study protocol was granted exemption from full review by the Institutional Review Board at the University of North Carolina Chapel Hill because it involved the use of existing, de-identified data.

\section{Results}

\section{Study population}

Five thousand and sixty-one subjects met inclusion criteria after exclusion of 180 due to the diagnosis of HCC or procedures used to treat HCC. Table 1 shows the demographic information for this cohort. Fifty-six percent were White, 35\% African American, 2\%

Native American, $0.5 \%$ Asian American, and 6\% other/unreported. A significant proportion of the sample lived in a rural county $(42 \%)$. Subjects were seen more often under acute-care settings than in outpatient clinic visits-70\% were seen at least once in an E.D. or were hospitalized. Forty-eight percent had one or more visits to a primary care physician (median visits 9 , mean 12 ) and only $21 \%$ were seen by a gastroenterologist (median visits 0 , mean 2 ).

Alcohol use or abuse diagnostic codes were identified in 59\%.[Table 2] Thirty percent had hepatitis $\mathrm{C}$, and the prevalence of other risk factors such as hemochromatosis, non-alcoholic steatohepatitis, primary biliary cirrhosis, hepatitis B, and alph-1 antitrypsin deficiency was less than $10 \%$. No identifiable risk factors were found in $24 \%$. HCC developed in $77(2 \%)$ over the time of the study. 


\section{Surveillance}

Of the 5,061 patients, only 1,268 (26\%) underwent at least one exam that could be used for HCC surveillance over 15 months. AFP testing alone was performed for an additional $4 \%$. When limited to ultrasound use, only $17 \%$ of the sample underwent imaging. Abdominal ultrasound was the most commonly ordered radiographic study (38\% of total imaging). CT and MRI were ordered in approximately equal frequencies (18\% and $16 \%$ respectively). Of those who received imaging, 743 (59\%) received just one imaging test, while 304 (24\%) had 2 and $221(17 \%)$ had $>2$. While not considered adequate for surveillance, AFP was performed at least once in $608(12 \%)$ patients.

\section{Variables associated with imaging}

In bivariate analysis, all factors save urban residence, race, Medicare dual-eligibility, and alpha-1 antitrypsin deficiency showed significant differences in imaging rates (data not shown). However, in multivariate analysis, female sex (O.R. 1.18, 95\% C.I. 1.02, 1.37), at least one episode of care in an academic center (O.R. 2.13, 95\% C.I. 1.82, 2.50), Medicare dual-eligibility (O.R. 1.69, 95\% C.I. 1.38, 2.07), viral related cirrhosis (O.R. 1.98, 95\% C.I. 1.67, 2.35), alcohol related cirrhosis (O.R. 1.20, 95\% C.I. 1.00, 1.47), and other cirrhosis with recognized risk factors (other chronic hepatitis or nonalcoholic liver disease, cholangitis, alpha-1 antitrypsin deficiency, primary biliary cirrhosis, or hemochromatosis; O.R. 2.16, 95\% C.I. 1.79, 2.6) were associated with increased odds of imaging.[Table 3] Subjects of Asian race had 2.22 times the odds of imaging; however, this result was not statistically significant $(95 \%$ C.I $0.90,5.5)$. At least one visit to a gastroenterologist (O.R. $2.81,95 \%$ C.I. $2.32,3.41)$ impacted the probability of imaging whereas visits to primary care did not (O.R. $0.94,95 \%$ C.I. $0.76,1.16)$. Advancing age was associated with a decreased probability of imaging $(\mathrm{p}<0.001)$.

\section{Subgroup Analyses}

We performed several analyses to examine utilization in various subgroups. Imaging utilization was similar when limited to patients under the age of 70 (4,488 subjects, $89 \%$ of the sample, $26 \%$ receiving imaging) and only slightly higher in subjects with at least 2 I.C.D.- 9 codes for cirrhosis (3,490 subjects, $69 \%$ of the sample, $28 \%$ receiving imaging) or at least 3 I.C.D.- 9 codes for cirrhosis (2,853 subjects, $59 \%$ of the sample, $31 \%$ receiving imaging). Fifty-seven percent were seen at least once in an E.D. and $47 \%$ were hospitalized. Of these, 1,103 (31\%) had abdominal imaging versus $185(12 \%)$ of those not hospitalized or seen in an E.D. $(p<0.0001)$. The percentage of subjects undergoing imaging was only $1 \%$ lower among those who did not develop HCC (4,984 subjects, 98\% of the sample, $25 \%$ receiving imaging). The factors found to significantly impact the probability of imaging in multivariable analysis persisted even when limited to subjects below the age of 70, subjects with two or three codes for cirrhosis, subjects who were not hospitalized or seen in an E.D, and among subjects who did not develop HCC save a few notable exceptions. Subjects with alcohol-related cirrhosis were slightly more likely to undergo imaging when limited to subjects under the age of 70 (O.R. 1.27, 95\% C.I. 1.04, 1.55) or to those with at least 2 or 3 codes for cirrhosis (O.R. 1.35, 95\% C.I. 1.06, 1.72 at least 2 codes; O.R. 1.39, 95\% C.I. $1.06,1.84$ at least 3 codes). For those not hospitalized or seen in an E.D., the age of the subject and the subject's sex was no longer an important factor. Also for patients seen only in non-urgent settings, viral hepatitis became the factor with the highest odds of undergoing imaging (O.R. 4.44, 95\% C.I. 2.81, 7.01), and being seen in a university setting had the next highest odds of undergoing imaging (O.R. 3.46, 95\% C.I. 2.29, 5.24).

When limited to ultrasound imaging alone, the same factors were found to be significant, though there were some differences in point estimates [Table 3]. One notable difference was in the odds of undergoing ultrasound imaging among Asian-Americans, which showed a 
non-significant decrease in the likelihood of undergoing an ultrasound (O.R. 0.59, 95\% C.I. $0.16,2.17)$.

\section{Discussion}

Medicaid benefits provide crucial coverage to persons with limited or no income, allowing access to preventive services that might otherwise be forsaken in favor of other necessities. However, utilization of preventive services such as Pap smear testing, mammograms, or colonoscopy is suboptimal.(14-16) Surveillance for HCC is no different, as we found that only one quarter of the NC Medicaid population underwent an exam that could be used for surveillance over a 15 -month observation period, falling to $12 \%$ for the 1520 patients who were only seen in outpatient settings. Ideally, all eligible patients should have undergone at least one imaging exam. While care in an academic center or seeing a gastroenterologist increased the odds of imaging, seeing a primary care provider did not.

Two prior studies of patients with HCC examined surveillance rates in VA and Medicare populations. Despite being older (average age 75 years), 38\% of the Medicare population had prior surveillance(9) compared to only $28 \%$ of VA patients with HCC.(8) Another study of VA hepatitis C cirrhotics without HCC yielded a rate of $42 \%$,(10) highlighting the potential differences in surveillance rates when patients with or without HCC are examined. However, all previous studies used data gathered before or during 2005 when the AASLD guidelines were first published. No studies have examined the Medicaid population. We found the imaging rate (which we took as a surrogate for surveillance rate) in the Medicaid population is one of the lowest reported at just $26 \%$. If we limit our analysis to just ultrasound exams, the AASLD recommended screening tool, the rate falls to $17 \%$.

There are multiple reasons for the poor utilization of surveillance even after publication of the AASLD guidelines. Barriers to healthcare utilization are likely greater in the Medicaid population. No studies have undertaken qualitative or quantitative research into patient barriers to HCC surveillance, but factors such as poverty, lack of transportation or loss of work hours have impacted screening for other diseases in Medicaid populations.(17) Others may simply not see the importance of surveillance, reflecting poor health literacy. Dubard and colleagues found that only approximately $50 \%$ of colorectal, breast and cervical cancer screening exams recommended by primary care providers were actually obtained in a similar NC Medicaid cohort.(14)

Provider attitudes play a crucial role as indicated by variation in surveillance rates for patients seen by primary care physicians versus gastroenterologists. Our subjects were seen twice as often by a primary care provider as compared to a gastroenterologist. However, those seen by a gastroenterologist were nearly three times as likely to undergo imaging, an effect that persisted among those patients only seen in the outpatient setting. The arguments for HCC surveillance may be compelling to some, but not all health care professionals.(18) Though endorsed by the AASLD and the European Association for the Study of the Liver (EASL), the National Cancer Institute Physician Data Query (NCI P.D.Q.()) panel does not support surveillance for HCC due to lack of evidence showing a clear mortality benefit.(19)

Alternatively, primary care providers may simply be unaware of surveillance guidelines. Delayed uptake of specialty guidelines by primary care has been seen in other best-practice scenarios.(20-24) Slow dissemination of best-practice guidelines may also explain why care at academic centers increased the odds of surveillance in our study and others.(9) Providers at academic centers have easier access to subspecialty conferences and continuing medical education opportunities, particularly in the field of hepatology. 
Unlike other studies that suggest urban locale is associated with surveillance, our study found no such association.(8) Ultrasound testing is available in most non-urban communities in our state, so lack of an independent association is plausible. Data on the association between alcohol abuse and surveillance are conflicting.(8) In our analysis, the effect of alcohol abuse did not persist among those seen only in the outpatient setting suggesting that the majority of exams ordered for alcoholic cirrhotics may have been for diagnostic purposes in the acute care setting. The decreased imaging rate with age may be explained by declining benefit of screening. This age effect did not persist in the outpatient setting suggesting that the hospitalized elderly may be more ill and unable to withstand HCC therapy thus obviating the need to screen.

Major strengths of our data include the large sample size and near-complete capture of billed services including those also covered by Medicare. Only $8 \%-12 \%$ of NC Medicaid patients are estimated to have supplemental non-Medicare insurance [personal communication January 2011, Tara Larson, Chief Clinical Operations Office, N.C. Department of Health and Human Services, (25)]. Even if all $12 \%$ covered by supplemental insurance underwent imaging not captured in our dataset, the imaging rate would have risen to only $38 \%$. We used ICD-9 codes for cirrhosis that have previously been shown to have high positive (90\%) and negative (87\%) predictive values for identifying patients with and without cirrhosis.(26) These codes were validated in a VA population. We were unable to confirm validity in this Medicaid cohort because the dataset contained only de-identified administrative data. To address this, we performed subgroup analysis limited to patients with at least 2 and 3 repeated codes for cirrhosis, and the imaging rates were essentially the same. Additionally, $80 \%$ of patients with a cirrhosis code also had another liver diagnosis or a complication of cirrhosis (e.g. hepatic encephalopathy, jaundice, ascites) suggesting misclassification bias was probably minimal. We also limited our cohort to those without HCC, and even exclusion of patients who developed HCC during the study period, did not change our results significantly.

A significant weakness in our study is that only $30 \%$ of the cohort was seen in the outpatient setting where health maintenance is typically addressed. Therefore our data probably reflects poor access or utilization of outpatient care by Medicaid patients more than lack of HCC surveillance by outpatient providers. Nevertheless, $30 \%$ of this large dataset still represents 1520 patients, and their imaging rate was less than $12 \%$. In any case, the imaging rate is abysmal, and our data highlight the challenges of providing preventive services to a cohort that does not routinely present for outpatient care. As the incidence of HCC cases rises overall, we will likely see mostly late-stage HCC in the Medicaid population due to the decreased opportunities to screen. Better access and utilization of outpatient care are clearly needed. For now, increased awareness of HCC surveillance guidelines by inpatient providers may represent an opportunity to detect earlier-stage tumors in Medicaid patients who often do not present for outpatient care without a compelling reason.

Another limitation of the data is the inability to differentiate between surveillance and diagnostic exams, even in the outpatient setting. The database we used is robust in diagnostic and procedural billing codes but lacks detailed clinical data such as clinical notes or laboratory results. One study has used an algorithm to distinguish between surveillance and diagnostic uses of AFP and ultrasound.(27) However, the algorithm used HIV status which was not available in this dataset, and the algorithm was less helpful for ultrasound compared to AFP. Practically speaking, any of the diagnostic imaging ascertained would be considered sufficient for HCC surveillance even if it were ordered for other purposes. The inability to identify the clinical intent of the imaging accomplished only biases our results toward overestimating the true surveillance rate. Clearly, the rate of surveillance is unacceptably low. 
Broad adoption of HCC surveillance may significantly reduce HCC mortality among cirrhotics. However, current data suggests the guidelines are followed sparingly and have had little penetration into non-gastroenterology practices for the Medicaid population. Few Medicaid cirrhotics even present for outpatient care, and lack of uptake by the primary care sector translates into very low surveillance rates overall. Much more must be done if the guidelines are to have community effectiveness in the Medicaid population. Identification of barriers to HCC surveillance among both patients and providers, recruitment of primary care providers, and improved access to subspecialty care will be necessary. Such penetration into the Medicaid population should not be dismissed as insurmountable. Ultrasound is available in most rural and urban communities. The exam is relatively quick, painless and without the discomfort that can be engendered by other screening exams such as pelvic exams, colonoscopies and mammograms. Therefore, mitigation of barriers to surveillance through establishment of educational and system interventions and improved utilization of outpatient services could greatly improve outcomes for Medicaid patients with cirrhosis.

\section{Acknowledgments}

The authors wish to acknowledge the contributions of Carol Q. Porter, M.S., who provided data abstraction and dataset preparation services through the Cecil G. Sheps Center for Health Services Research, University of North Carolina, Chapel Hill, North Carolina.

\section{Source of Funding:}

The authors received an unrestricted grant from Onyx pharmaceuticals (Applied to data usage and programming costs. The funding agency had no part in the design and conduct of the study; analysis and interpretation of the data; or preparation, review, or approval of the manuscript). Dr. Palmer was supported in part by a T32 National Institutes of Health Training Grant 5-T32 DK007634 (supported author's time to perform all aspects of research). Dr. Kappelman was supported in part by the National Center for Research Resources Grant KL2 RR025746 and the National Institute for Diabetes and Digestive and Kidney Diseases Grant P30 DK034987 (supported author's time to contribute to the design and conduct of the study, analysis and interpretation of the data, and review and approval of the manuscript).

Guarantor of the article: Dr. Palmer is the guarantor of the article and takes full responsibility for the conduct of the study.

\section{References}

1. Minino AM, Heron MP, Murphy SL, et al. Deaths: final data for 2004. Natl Vital Stat Rep. 2007; 55:1-119. [PubMed: 17867520]

2. Shaheen NJ, Hansen RA, Morgan DR, et al. The Burden of Gastrointestinal and Liver Diseases, 2006. The American Journal of Gastroenterology. 2006; 101:2128-2138. [PubMed: 16848807]

3. Bruix J, Sherman M. Management of hepatocellular carcinoma. Hepatology. 2005; 42:1208-1236. [PubMed: 16250051]

4. Kanwal F, Hoang T, Kramer JR, et al. Increasing Prevalence of HCC and Cirrhosis in Patients With Chronic Hepatitis C Virus Infection. Gastroenterology. 2011; 140:1182-1188 e1. [PubMed: 21184757]

5. El-Serag HB, Rudolph KL. Hepatocellular Carcinoma: Epidemiology and Molecular Carcinogenesis. Gastroenterology. 2007; 132:2557-2576. [PubMed: 17570226]

6. Zhang B-H, Yang B-H, Tang Z-Y. Randomized controlled trial of screening for hepatocellular carcinoma. Journal of Cancer Research and Clinical Oncology. 2004; 130:417-422. [PubMed: 15042359]

7. Chalasani N, Said A, Ness R, et al. Screening for hepatocellular carcinoma in patients with cirrhosis in the United States: results of a national survey. Am J Gastroenterol. 1999; 94:2224-9. [PubMed: 10445554]

8. Davila JA, Weston A, Smalley W, et al. Utilization of screening for hepatocellular carcinoma in the United States. J Clin Gastroenterol. 2007; 41:777-82. [PubMed: 17700427] 
9. Davila JA, Morgan RO, Richardson PA, et al. Use of surveillance for hepatocellular carcinoma among patients with cirrhosis in the United States. Hepatology. 2010; 52:132-141. [PubMed: 20578139]

10. Davila JA, Henderson L, Kramer JR, et al. Utilization of Surveillance for Hepatocellular Carcinoma Among Hepatitis C Virus-Infected Veterans in the United States. Annals of Internal Medicine. 2011; 154:85-93. [PubMed: 21242365]

11. Wong GL, Xu HX, Xie XY. Detection of focal liver lesions in cirrhotic liver using contrastenhanced ultrasound. World J Radiol. 2009; 1:25-36. [PubMed: 21160718]

12. Rural County Map. Copyright 2000-2010. [cited March 25, 2011]; Available from: http:// www.ncruralcenter.org/rural-county-ma.html

13. Deyo RA, Cherkin DC, Ciol MA. Adapting a clinical comorbidity index for use with ICD-9-CM administrative databases. Journal of Clinical Epidemiology. 1992; 45:613-619. [PubMed: 1607900]

14. DuBard CA, Schmid D, Yow A, et al. Recommendation for and Receipt of Cancer Screenings Among Medicaid Recipients 50 Years and Older. Arch Intern Med. 2008; 168:2014-2021. [PubMed: 18852404]

15. Peterson NB, Han J, Freund KM. Inadequate follow-up for abnormal Pap smears in an urban population. J Natl Med Assoc. 2003; 95:825-32. [PubMed: 14527050]

16. Guessous I, Dash C, Lapin P, et al. Colorectal cancer screening barriers and facilitators in older persons. Preventive Medicine. 2010; 50:3-10. [PubMed: 20006644]

17. Mastroberti M, Stein JE. Barriers to timely mammography. HMO Pract. 1996; 10:104-7. [PubMed: 10160284]

18. Lederle FA, Pocha C. Screening for Liver Cancer: The Rush to Judgment. Annals of Internal Medicine. 2012; 156:387-389. [PubMed: 22393134]

19. Physician Data Query® Panel. Liver (Hepatocellular) Cancer Screening (PDQ®). 2008. [cited 2008 November 26, 2008]; Available from: http://www.cancer.gov/cancertopics/pdq/screening/ hepatocellular/healthprofessional

20. Harrold LR, Field TS, Gurwitz JH. Knowledge, patterns of care, and outcomes of care for generalists and specialists. J Gen Intern Med. 1999; 14:499-511. [PubMed: 10491236]

21. De Smet BD, Fendrick AM, Stevenson JG, et al. Over and under-utilization of cyclooxygenase-2 selective inhibitors by primary care physicians and specialists: the tortoise and the hare revisited. $\mathrm{J}$ Gen Intern Med. 2006; 21:694-7. [PubMed: 16808768]

22. Hirth RA, Fendrick AM, Chernew ME. Specialist and Generalist Physicians' Adoption of Antibiotic Therapy to Eradicate Helicobacter pylori Infection. Medical Care. 1996; 34:1199-1204. [PubMed: 8962586]

23. Landon BE, Wilson IB, Cohn SE, et al. Physician specialization and antiretroviral therapy for HIV. J Gen Intern Med. 2003; 18:233-41. [PubMed: 12709089]

24. Rappaport KM, Forrest CB, Holtzman NA. Adoption of Liquid-Based Cervical Cancer Screening Tests by Family Physicians and Gynecologists. Health Services Research. 2004; 39:927-948. [PubMed: 15230935]

25. US Government Accountability Office. GAO-06-862 MEDICAID THIRD-PARTY LIABILITY: Federal Guidance Needed to Help States Address Continuing Problems. US Government Accountability Office. , editor. Washington, D.C.: 2006.

26. Kramer JR, Davila JA, Miller ED, et al. The validity of viral hepatitis and chronic liver disease diagnoses in Veterans Affairs administrative databases. Alimentary Pharmacology \& Therapeutics. 2008; 27:274-282. [PubMed: 17996017]

27. Richardson P, Henderson L, Davila J, et al. Surveillance for Hepatocellular Carcinoma: Development and Validation of an Algorithm to Classify Tests in Administrative and Laboratory Data. Digestive Diseases and Sciences. 2010; 55:3241-3251. [PubMed: 20844957] 
Table 1

Characteristics of NC Medicaid subjects with cirrhosis, 2006-2007 ${ }^{a}$

\begin{tabular}{|c|c|c|c|c|}
\hline & \multicolumn{2}{|c|}{ Total } & \multicolumn{2}{|c|}{ Received Imaging } \\
\hline & $\stackrel{N}{N}$ & $\begin{array}{c}\text { Percent } \\
(\%)^{*}\end{array}$ & $\mathbf{N}$ & $\begin{array}{c}\text { Percent } \\
(\%)^{\dagger}\end{array}$ \\
\hline Age & $54(12)^{t}$ & - & - & - \\
\hline Sex (female) & 2,220 & (44) & 629 & (27) \\
\hline \multicolumn{5}{|l|}{ Race } \\
\hline White & 2,841 & (56) & 740 & (26) \\
\hline African American & 1,780 & (35) & 442 & (25) \\
\hline Native American & 91 & (2) & 21 & (23) \\
\hline Asian American & 27 & $(<1)$ & 11 & (41) \\
\hline Other/Unreported & 322 & (6) & 81 & (25) \\
\hline Seen in an E.D. ${ }^{\S}$ & 2,885 & (57) & 911 & $(31)$ \\
\hline Hospitalized & 2,379 & (47) & 876 & (37) \\
\hline Primary Care Visit(s) & 2,429 & (48) & 730 & $(30)$ \\
\hline Gastroenterology Visit(s) & 1,063 & (21) & 503 & (47) \\
\hline Academic Center Care & 1,113 & (22) & 504 & (46) \\
\hline Urban Residence & 2,958 & (58) & 762 & (26) \\
\hline Medicare Dual-Eligibility & 2,659 & (53) & 651 & $(50)$ \\
\hline
\end{tabular}

${ }^{a}$ All data were derived from North Carolina Cost and Quality Initiative database for 2006-2007. The study population included all individuals over the age of 18 with at least one recording of I.C.D.-9 code 571.2 (alcoholic cirrhosis of liver) or I.C.D.-9 code 571.5 (cirrhosis of liver without mention of alcohol) and at least 15 months of continuous Medicaid enrollment during 2006-2007.

Percent of total sample $(5,061)$

${ }^{\dagger}$ Percent of row total

Ftandard deviation

$\xi_{\text {E.D. }}=$ emergency department 
Table 2

Cirrhosis Risk Factors

\begin{tabular}{|c|c|c|c|c|}
\hline & \multicolumn{2}{|c|}{ Total } & \multicolumn{2}{|c|}{ Received Imaging } \\
\hline & $\underset{(N=5,061)}{N}$ & $\begin{array}{l}\text { Percent } \\
(\%)^{*}\end{array}$ & $\mathbf{N}$ & $\begin{array}{c}\text { Percent } \\
(\%)^{\dagger}\end{array}$ \\
\hline Alcohol related & 2,991 & (59) & 821 & (27) \\
\hline Hepatitis C & 1,493 & (30) & 615 & (41) \\
\hline Hepatitis B & 205 & (4) & 100 & (49) \\
\hline Hemochromatosis & 359 & (7) & 138 & (38) \\
\hline $\begin{array}{l}\text { Alph-1 antitrypsin } \\
\text { deficiency }\end{array}$ & 5 & $(0.1)$ & 1 & (20) \\
\hline Primary biliary cirrhosis & 79 & (2) & 42 & (53) \\
\hline Cholangitis & 32 & $(0.6)$ & 27 & (85) \\
\hline $\begin{array}{l}\text { Other chronic active } \\
\text { hepatitis/ non-alcoholic } \\
\text { liver disease }\end{array}$ & 400 & (8) & 220 & (57) \\
\hline Unknown & 1,207 & (24) & 170 & (14) \\
\hline
\end{tabular}


Table 3

Multivariable logistic regression model of factors associated with imaging ${ }^{a}$

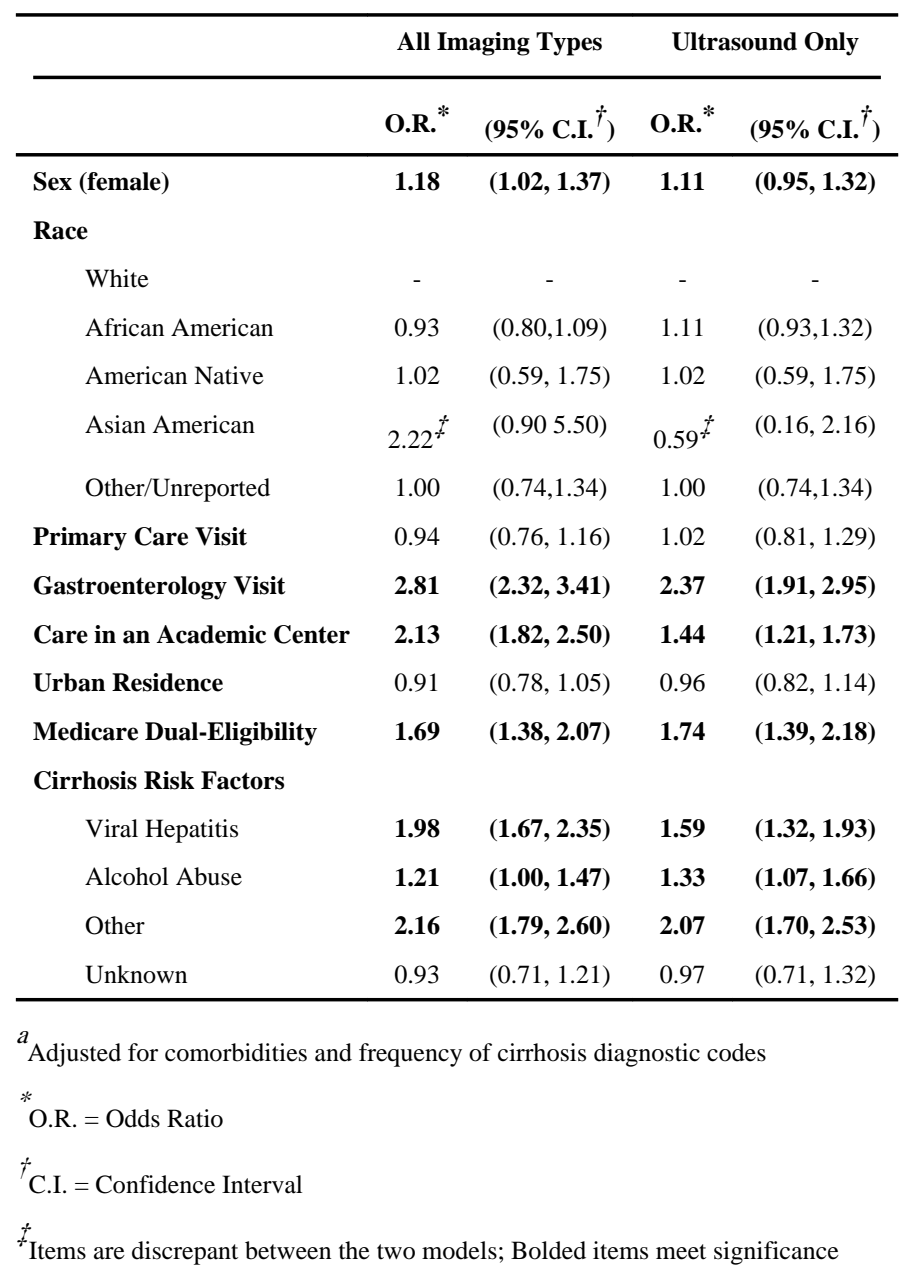

J Clin Gastroenterol. Author manuscript; available in PMC 2014 September 01. 